\title{
Incidence of secondary atrophic rhinitis following endoscopic sinonasal tumour surgery: a retrospective
} review*

\section{Mogammad S. Kamedien, Taseer F. Din, Darlene Lubbe}

Division of Otolaryngology, University of Cape Town, Cape Town, Western Cape, South Africa
Rhinology Online, Vol 3: 100 - 105, 2020

http://doi.org/10.4193/RHINOL/20.034

*Received for publication:

April 21, 2020

Accepted: June 12, 2020

Published: July 22, 2020

\begin{abstract}
Background: Sinonasal tumours have been resected endoscopically at Groote Schuur Hospital, Cape Town, since 2003. The surgery, although called "minimally invasive" is often very aggressive and destructive to the nasal structures. The removal of nasal turbinates has always been considered sacrilege due to the alleged risk of causing atrophic rhinitis. If the theory that atrophic rhinitis follows a simple turbinectomy is indeed true, one would expect all patients undergoing radical resection of the nasal structures to develop atrophic rhinitis. This has not been our experience.
\end{abstract}

Methods: This retrospective case review includes all patients with both benign and malignant sinonasal tumours that were endoscopically resected by a single surgeon from 2006 - 2010 at our institution. We recorded the incidence of postoperative atrophic rhinitis over a 2-year follow-up period.

Results: 51 patients (34M:17F) were included in the study. Patients with residual or recurrent tumour ( $n=19)$ and patients who had received adjuvant radiotherapy $(n=17)$ had a statistically significant risk of developing symptoms and signs suggestive of atrophic rhinitis. Variables such as age, gender, extent of surgery, bilateral disease, and benign/malignant tumour were not statistically significant in the development of symptoms and signs suggestive of atrophic rhinitis.

Conclusions: The extent of endoscopic tumour resections and turbinectomies, even in the presence of roomy nasal cavities, did not predispose to AR. Residual or recurrent disease and those who receive adjuvant radiotherapy have a statistically increased chance of developing symptoms and signs consistent with AR over time. Such patients require careful endoscopic examination to remove crusts and to exclude residual/recurrent tumour or exposed bone deep to the crusts.

Key words: atrophic rhinitis, endoscopic resection, sinonasal tumours, medial maxillectomy, turbinectomy

\section{Introduction}

Atrophic Rhinitis (AR) is a chronic debilitating disease of the nasal passages that is characterized by progressive atrophy of the nasal mucosa, nasal crusting, fetor (foul smelling nasal discharge) and enlargement of the nasal space with paradoxical subjective nasal congestion. Patients with AR may also complain of a disordered sense of smell, but complete anosmia is rare until late in the disease process ${ }^{(1)}$. Much confusion exists in the literature regarding the diagnosis, aetiologic factors, and treatment of AR. Different terminologies have been used in the literature (atrophic rhinitis, rhinitis sicca/dry nose, ozaena and empty nose syndrome $\left.{ }^{(2,3)}\right)$ and this has made investigation of the causes and treatment of the condition difficult.

Since the middle of the 20th century, various authors have divided AR into two separate entities. The distinction between the two diseases lie in the aetiology. Primary atrophic rhinitis (PAR) is of spontaneous onset, progresses slowly and has an unspecified aetiology. Secondary atrophic rhinitis (SAR) develops after chronic rhinosinusitis, chronic granulomatous disease, 
excessively aggressive surgery in inflammatory/infectious nasal and sinus surgery, nasal trauma or irradiation. PAR may represent early ozaena before the submucosal destructive processes brought on by inheritable or infections causes have progressed to their end state ${ }^{(4)}$. SAR is much more commonly encountered, although it is not more completely understood. Characteristic findings in both forms include nasal crusting, enlarged nasal cavities, resorption of the turbinates, mucosal atrophy, and paradoxical nasal congestion ${ }^{(5)}$.

There have been numerous debates about whether there is an association between SAR and modified or total reductive turbinate surgery ${ }^{(6-15)}$. If the theory about developing AR after turbinectomy is true, one would expect all patients undergoing radical resection of the nasal structures to develop AR. Our study explores whether radical resection of the nasal structures did indeed cause SAR .

\section{Materials and Methods}

\section{Study population}

All patients with sinonasal tumours (benign or malignant) who had endoscopic resection by the 3rd author (DL) at Groote Schuur Hospital over a five-year period (2006-2010) were eligible for inclusion. Patients required at least two years' follow-up.

\section{Study design}

A retrospective case review was done on the study population above. All patients included in the study were given standard postoperative instructions and were followed up for at least two years after surgery. They were assessed for nasal obstruction, discharge and crusting at each visit.

The same surgeon (DL) assessed all patients preoperatively. A thorough history and examination, including nasendoscopy was performed prior to surgery. Special investigations included a histological diagnosis and imaging (CT and/or MRI of the sinuses). Tumour location and extent of surgery were documented and patients were regularly followed up. Patients were given instructions on postoperative care about nasal douching with normal saline twice daily. Postoperative follow-up was done at the following intervals: 2 weeks, 6 weeks, 3 months, 6 months, 12 months, 18 months, and 24 months, or sooner at the discretion of the surgeon. Patients were assessed for nasal obstruction, crusting and discharge suggestive of a diagnosis of AR at each visit.

\section{Statistical analysis}

Incidence proportions, mean and median values were calculated for all patient characteristics, as appropriate. Relative ratios, p-values and 95\% confidence intervals were used to estimate the relative risk of developing the outcome of interest with respect to various categorical predictors by generalized linear regression models at 12 weeks. The statistical significance of the association between outcome and exposure over the seven time-periods was assessed by the xtreg (cross-sectional time dependent regression) procedure. Stata 12 software (StataCorp, College Station, TX, USA) was used to carry out all analyses and results interpreted with the help of a statistician.

\section{Results}

Fifty-one patients (34 males, 17 females) had sinonasal tumours endoscopically resected between 2006 and 2010 that met the inclusion criteria. Mean patient age was 51.1 years (range 13-78 years); this was normally distributed.

Eighteen patients had malignant tumours and 33 patients had benign lesions (Figure 1). Nineteen malignancies were T1, 12 were T2, 2 were T3 and 18 were T4. Forty-two malignancies had surgery with curative intent, and 9 had extensive tumours treated by palliative debulking and adjuvant treatment (Figure 1). The only medical treatment throughout the postoperative course was normal saline nasal douches twice daily.

Criteria used to define SAR were nasal obstruction and nasal discharge together with clinical findings of nasal discharge/ excessive crusting and a wide nasal cavity. During follow-up, biopsies of areas abnormal mucosa were taken and sent for microbiological and histopathological examination. No pus swabs were taken. Potential recall bias was minimal as the symptoms and clinical signs seen by the same clinician were documented in the notes. As was to be expected, all patients reported nasal obstruction and nasal discharge at the initial postoperative visit, as well as signs of nasal discharge and crusting. These findings were also evident in most patients at the 6 weeks follow-up despite proper nasal hygiene. At 12 weeks postoperatively, there was a significant reduction in nasal symptoms and signs.

However, this reduction in nasal symptoms and signs after 12 weeks did not apply to patients who had residual or recurrent sinonasal malignancy. The incidence proportion (IP) for developing nasal crusting and discharge consistent with AR in those with residual/recurrent malignancy $(n=19)$ relative to those with complete resection of tumour $(n=32)$ at the 12 weeks turning point was $78.9 \%$ and $3.1 \%$ respectively, with a relative risk (RR) of $25.3,95 \%$ confidence interval $(\mathrm{Cl})$ of $3.7-179.6$ at week 12 . Comparing the IPs over time in these two groups resulted in an overall $p$-value $<0.001$, where this difference was maintained up to the 2-year follow-up period. Thus, patients with residual/ recurrent tumour developed symptoms and signs consistent with a diagnosis of AR at 12-weeks postoperatively; this was maintained over time, relative to those in whom the tumour was completely excised (Comparative Graphs 1 \& 2 / Table 1). 


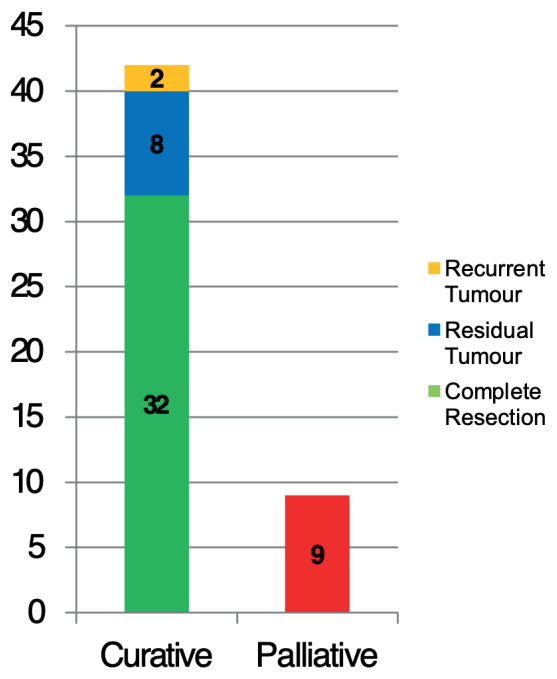

\begin{tabular}{|c|c|}
\hline $\mathbf{N}$ & Tumour \\
\hline 2 & Squamous Cell Ca \\
\hline 1 & Adenoid Cystic Ca \\
\hline 1 & Esthesioneuroblastoma \\
\hline 1 & Hemangiopericytoma \\
\hline 1 & Meningioma \\
\hline 1 & Ossifying Fibroma \\
\hline 1 & Osteoclastoma \\
\hline 1 & Schwannoma \\
\hline 2 & Inverted Papilloma \\
\hline 2 & Squamous Cell Ca \\
\hline 2 & Sinonasal Melanoma \\
\hline 1 & Adenoid Cystic Ca \\
\hline 1 & Esthesioneuroblastoma \\
\hline 1 & Ossifying Fibroma \\
\hline 1 & Sinonasal Adeno Ca \\
\hline 20 & Inverted Papilloma \\
\hline 2 & Benign Nasal Mass \\
\hline 2 & Neuroendocrine Ca \\
\hline 1 & Adenoid Cystic Ca \\
\hline 1 & Chondrosarcoma \\
\hline 1 & Fibrous Dysplasia \\
\hline 1 & Hemangiopericytoma \\
\hline 1 & Ossifying Fibroma \\
\hline 1 & Sinonasal Carcinoid \\
\hline & Sinonasal Melanoma \\
\hline 1 & Squamous Cell Ca \\
\hline 1 & \\
\hline 1 & \\
\hline 1 & \\
\hline 1 & \\
\hline 1 & \\
\hline 1 &
\end{tabular}

Figure 1. Curative Surgery ( $n=42)$, Palliative Debulking $(n=9), 2$ years post-operative.

We next investigated patients who had an inferior turbinectomy because of tumor involvement of the inferior turbinate (IT) or as part of an endoscopic medial maxillectomy procedure $(n=38)$, and compared them to patients who had the IT preserved $(\mathrm{n}=13)$. The IP for developing symptoms and signs consistent with AR in those who had the IT resected relative to those who had the IT preserved at the 12 weeks turning point was $31.6 \%$ and $30.8 \%$, respectively. This results in a RR of $1.0,95 \% \mathrm{Cl} 0.4$ -2.63 at week 12. Comparing the IPs over the 2-year follow-up between these 2 groups demonstrated an overall $p$-value $=$ 0.96 , which is not statistically significant. Thus, resecting the IT was not found to be a factor in the development of AR; this was maintained over time (Comparative Graphs 3 \& 4 / Table 1).

We also looked at the patients who had both inferior (IT) and middle turbinates (MT) resected $(n=35)$ and compared them to patients who had the IT and MT preserved $(n=10)$. The IP for developing symptoms and signs consistent with a diagnosis of $A R$ in those who had both IT and MT resected relative to those who had the IT and MT preserved at the turning point of 12 weeks were $34.3 \%$ and $40.0 \%$, respectively. This resulted in a RR of $0.9,95 \% \mathrm{Cl}$ of $0.5-3.6$ at 12 weeks. Comparing the IPs over the 2-year follow-up between these 2 groups resulted in an overall p-value of 0.75 which was not statistically significant. Thus, resecting both the IT and MT did not influence the development of AR over time (Comparative Graphs 5 \& 6 / Table 1).
We next compared patients who received adjuvant radiotherapy $(n=17)$ with those who did not $(n=34)$. The IP for developing symptoms and signs consistent with a diagnosis of AR at the turning point of 12 weeks in those who had adjuvant radiotherapy relative to those we did not, was $47.1 \%$ and $23.5 \%$, respectively. This resulted in a RR of $2.0,95 \% \mathrm{Cl} 0.9-4.4$ at week 12. Even though this was not statistically significant at this point, comparing the IPs over 2-year follow-up between the two groups resulted in an overall $p$-value $<0.001$, suggesting that patients who received adjuvant radiotherapy develop symptoms and signs consistent with AR at 12 weeks postoperatively and that this was maintained over time relative to those who did not receive adjuvant radiotherapy.

Variables such as age, gender, bilateral disease and whether tumour was benign or malignant or extent of surgery were not statistically significant in the development of AR over time.

\section{Discussion}

There has been a rapid evolution of the capabilities of endoscopic sinus surgeons, as well as advances in endoscopic instrumentation and intraoperative imaging, and experience with endoscopic repair of even large skull base defects. This has opened new and exciting surgical possibilities. Initially advocated for obstructive inflammatory disease, endoscopic approaches are now being increasingly used to resect nasal and paranasal 


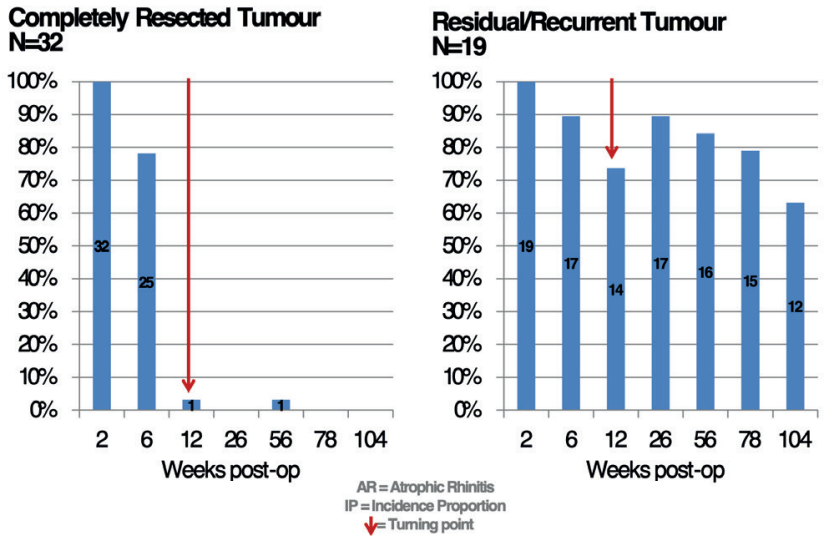

Comparative Graphs 1 \& 2. IP of patients with symptoms and signs suggestive of AR.

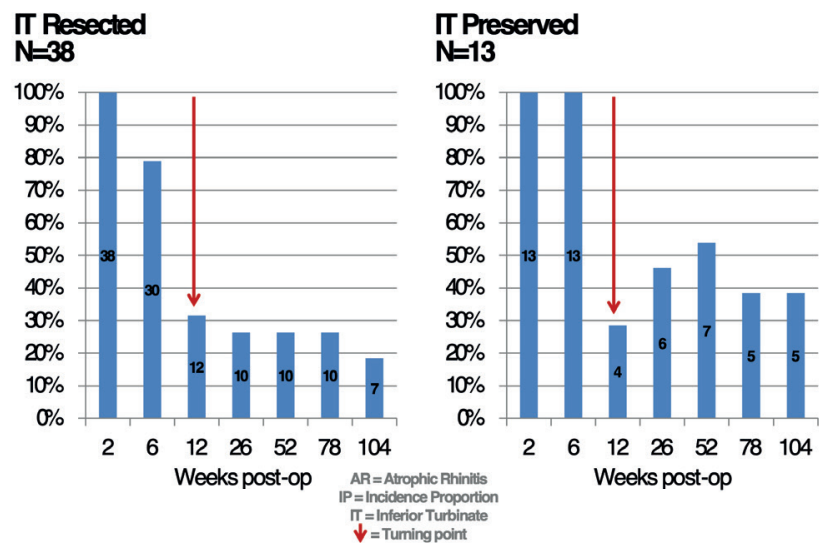

Comparative Graphs 3 \& 4. IP of patients with symptoms and signs suggestive of $A R$.

sinus tumours, previously resected through more traditional (transfacial or craniofacial) approaches. Endoscopic resection of benign lesions and some malignant lesions reflect a fundamental change from traditional therapeutic concepts and modalities $^{(1-3)}$. Almost all benign and intermediate sinonasal tumours have been resected endoscopically at Groote Schuur Hospital in Cape Town, South Africa since 2003 using a minimally invasive surgical approach. Although called "minimally invasive" because no external incisions are made, resection of nasal structures is often extensive surgery.

Current literature suggests that one of the causes of SAR is overly aggressive nasal surgery, particularly turbinate surgery ${ }^{(6-9)}$. If the theory that AR develops after a simple turbinectomy is correct, one would expect all/most patients undergoing radical resection of the nasal structures to develop AR. This has not been the case in our study as was shown by our results.

A number of studies have examined the likely aetiology and diagnosis of SAR. In some studies done between 1987 and 1999, authors report $15-89 \%$ of their own patients experiencing postoperative AR symptoms after nasal tissue removal, especially

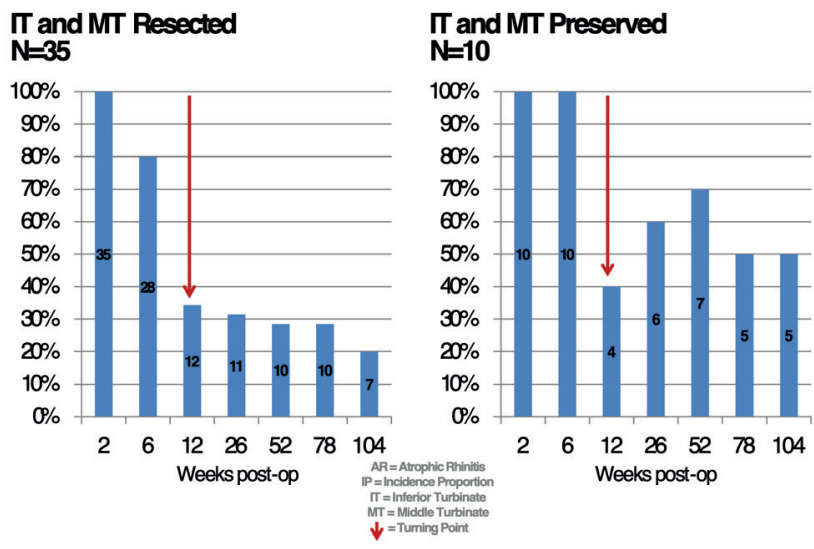

Comparative Graphs 5 \& 6. IP of patients with symptoms and signs suggestive of AR.

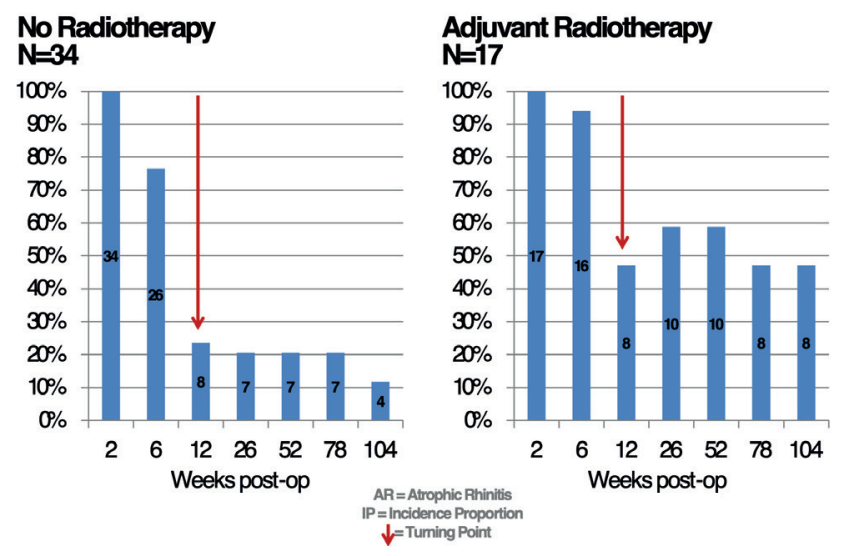

Comparative Graphs 7 \& 8. IP of patients with symptoms and signs suggestive of AR.

following turbinectomy, while other authors report that they never encountered AR after turbinectomy ${ }^{(10-15)}$. An extensive literature review is presented in Table 2 . There are no reports of AR following endoscopic sinonasal tumour surgery or surgery for inverted papillomas in the English literature.

Based on our study, symptoms of nasal obstruction and nasal discharge, and clinical signs of nasal discharge/excessive crusting blockage tend to settle by 12 weeks postoperatively. This possibly reflects the time required for re-epithelialisation to occur, although further studies are required to be done to verify this reasoning.

In our experience, patients did not manifest symptoms of AR 12 weeks following extensive endoscopic tumour resections and after turbinectomies, even in the presence of roomy nasal cavities after turbinectomies. However, patients with residual/ recurrent sinonasal tumours and exposed bone have areas with disordered mucociliary clearance that do cause crusting and AR symptoms. Therefore, it is important to evaluate and follow patients with persistent nasal symptoms. Such patients require 
Table 1. Evaluation of relative risk of atrophic rhinitis (12 weeks post - op) and overall p-values.

\begin{tabular}{|c|c|c|c|c|c|}
\hline Variable & & $\mathbf{N}$ & $\%$ AR at 12 weeks & $\begin{array}{c}\text { Risk ratio }(95 \% \mathrm{Cl}) \text { at } \\
12 \text { weeks }\end{array}$ & $\begin{array}{l}\text { Overall p-value for } \\
\text { IP over the } 7 \text { time } \\
\text { periods }\end{array}$ \\
\hline \multirow{2}{*}{ Tumour } & Complete excision & 32 & 3.1 & 1.0 (ref) & \multirow{2}{*}{$<0.001$} \\
\hline & $\begin{array}{c}\text { Residual/Recurrent } \\
\text { disease }\end{array}$ & 19 & 78.9 & $25.3(3.6-176.3)$ & \\
\hline \multirow{2}{*}{ IT } & Preserved & 13 & 30.8 & 1.0 (ref) & \multirow{2}{*}{$=0.96$} \\
\hline & Resected & 38 & 31.6 & $1.0(0.4-2.63)$ & \\
\hline \multirow{2}{*}{ IT \& MT } & Preserved & 10 & 40.0 & 1.0 (ref) & \multirow{2}{*}{$=0.75$} \\
\hline & Resected & 35 & 34.3 & $0.9(0.5-3.6)$ & \\
\hline \multirow{2}{*}{$\begin{array}{l}\text { Adjuvant } \\
\text { Radiotherapy }\end{array}$} & No & 34 & 23.5 & 1.0 (ref) & \multirow{2}{*}{$<0.001$} \\
\hline & Yes & 17 & 47.1 & $2.0(0.8-4.4)$ & \\
\hline
\end{tabular}

$\mathrm{IT}$, inferior turbinate; MT, middle turbinate; AR, atrophic rhinitis; IP, incidence proportion.

Table 2. Incidence of AR correlated to extent of surgical resection.

\begin{tabular}{|c|c|c|c|}
\hline Study & $\mathbf{n}$ & Extent of surgical resection & Incidence of AR \\
\hline \multirow[t]{4}{*}{ Moore et al, $2001^{(4)}$} & 197 & Complete inferior and middle turbinectomy & $47(24 \%)$ \\
\hline & & Partial inferior and/or middle turbinectomy & $110(56 \%)$ \\
\hline & & ESS without turbinectomy & $20(10 \%)$ \\
\hline & & Partial maxillectomy for tumour & $12(6 \%)$ \\
\hline Moore et al, $1985^{(5)}$ & 18 & Total inferior turbinectomy & $16(89 \%)$ \\
\hline Warwick-Brown et al, $1987^{(6)}$ & 24 & Partial inferior turbinectomy & $18(75 \%)$ \\
\hline Salam et al, $1993^{(7)}$ & 25 & Total inferior turbinectomy & $4(16 \%)$ \\
\hline Oburra et al, $1995^{(8)}$ & 34 & Total inferior turbinectomy (Bilat) & $5(15 \%)$ \\
\hline Passali et al, $1999^{(9)}$ & 45 & Total inferior turbinectomy & $10(22 \%)$ \\
\hline Martinez et al, $1993^{(10)}$ & 29 & Total inferior turbinectomy & $1(3 \%)$ \\
\hline Odetoyinbo et al, $1987^{(11)}$ & 39 & Total inferior turbinectomy & $0(0 \%)$ \\
\hline Courtiss et al, $1999^{(12)}$ & 25 & Total inferior turbinectomy & $0(0 \%)$ \\
\hline Ophir et al, $1999^{(13)}$ & 38 & Total inferior turbinectomy & $0(0 \%)$ \\
\hline Cook et al, $1995^{(14)}$ & 31 & Partial middle turbinectomy & $0(0 \%)$ \\
\hline Talmon et al, $2000^{(15)}$ & 357 & Total inferior turbinectomy & $0(0 \%)$ \\
\hline
\end{tabular}

careful endoscopic examination to remove crusts and to exclude residual/recurrent tumour or exposed bone deep to the crusts.

Adjuvant radiotherapy was associated with symptoms and signs consistent with AR at 12 weeks postoperatively and that this was maintained over time relative to those who did not receive adjuvant radiotherapy. This is possibly due to the long-term side effects of radiotherapy on tissues and bone such as mucosal damage, destroying ciliary function, longstanding mucositis and radiogenic osteitis of the underlying bone. Further studies are needed to evaluate this (Comparative Graphs 7 \& 8 / Table 1).
Comparing a nasal cavity after tumour resection to one with empty nose syndrome also brings to attention some interesting hypotheses. After tumour resection, nasal cavities without residual/recurrent tumour, once de-crusted, are widely patent for nasal airflow. Symptoms and signs consistent with AR in such patients, in our experience, improves with time. Nasal cavities with the classic empty nose syndrome after turbinate surgery, are comparatively less wide; yet, these patients often have worse subjective complaints. One may ask - apart from aberrations in neurosensory systems resulting from improper healing in these nasal cavities associated with this syndrome(21), whether there is a psychological aspect to these patients, worth 
investigating?

\section{Limitations}

Our study contained a variety of histologies and therefore a spectrum of tumor behavioral factors.

\section{Conclusions}

From our series of patients, the extent of endoscopic tumour resections and turbinectomies, even in the presence of roomy nasal cavities, did not predispose to AR. We did however show that residual or recurrent disease and those who received adjuvant radiotherapy had a statistically increased chance of developing symptoms and signs consistent with AR over time. Such patients require careful endoscopic examination to remove crusts and to exclude residual/recurrent tumour or exposed bone deep to the crusts.

\section{Acknowledgments}

The authors thank all patients involved in the study, as well as Mr. Henri Carrara our statistician.

\section{Authorship contribution}

MSK wrote the protocol and collected the data. TFD, MSK and DL analysed, edited and approved the final manuscript.

\section{Conflict of interest}

No conflicts of interests to declare.

\section{Ethics approval and consent to participate} Not applicable.

\section{Consent for publication}

Not applicable.

\section{Availability of data and materials}

Not applicable.

\section{Funding}

None.

\section{References}

1. Banhiran W, Casiano RR. Endoscopic sinus surgery for benign and malignant nasal and sinus neoplasm. Curr Opin Otolaryngo Head Neck Surg. 2005;13:50-54

2. Parida PK, Gupta AK. Medial maxillectomy: A comparitive study as a surgical procedure. Otolaryngol Head Neck Surg. 2008;138:192199.

3. Busquets JM, Hwang PH. Endoscopic Resection of Sinonasal Inverted Papilloma: A Meta-Analysis. Otolaryngol Head Neck Surg. 2006;134:476-482

4. Moore EJ, Eugene B. Atrophic Rhinitis: A Review of 242 Cases. Am J Rhinol. 2001 Nov-Dec;15(6):355-361

5. Moore GF, Yonkers AJ, Freeman TJ, Ogren FP. Extended follow-up of total inferior turbinate resection for relief of chronic nasal obstruction. Laryngoscope. 1985;95:10951099

6. Warwick-Brown NP, Marks NJ. Turbinate Surgery: How Effective Is It? Otorhinolaryngology. 1987;49:314-320.

7. Salam MA, Wengraf C. Concho-antropexy or total inferior turbinectomy for hypertrophy of inferior turbinates? A prospective randomized study. J Laryngol Otol. 1993 Dec 107:1125-1128

8. Oburra HO. Complications following bilateral turbinectomy. East Afr Med J. 1995 Feb;72(2):101-102.

9. Passali D, Lauriello M, Anselmi M, Belluss
L. Treatment of hypertrophy of the inferior turbinate: Long term results in 382 patients randomly assigned to therapy. Ann Otol Rhinol Laryngol. 1999;108:569-575.

10. Martinez SA, Nissen AJ, Stock CR, Tesmer T. Nasal turbinate resection for relief of nasal obstruction. Laryngoscope. 1983 July;93: 871-875.

11. Odetoyinbo O. Complications following total inferior turbinectomy: facts or myths? Clin Otolaryngol. 1987;12:361-363.

12. Courtiss EH, Goldwyn RM. Resection of Obstructing Inferior Nasal Turbinates: A 10-year Follow Up. Plast Reconstr Surg. 1990;86:152-154.

13. Ophir D. Resection of Obstructing Inferior Turbinates Following Rhinoplasty. Plast Reconstr Surg. 1990 May; 85:724-727.

14. Cook PR, Begegni A, Bryant WC, Davis WE. Effect of partial middle urbinectomy on nasal airflow and resistance. Otolaryngol Head Neck Surg.1995 Oct;113(4):413-419.

15. Talmon Y, Samet A, Gilbey P. Total Inferior Turbinectomy: Operative Results and Technique. Ann Otol Rhinol Laryngol. 2000; 109:1117-1119.

16. Mackle T, Chambon G, Garrel R, Meieff M, Crampette L. Endoscopic treatment of sinonasal papilloma: a 12 year review. Acta Oto-Laryngologica 2008;128:670-674.

17. Harvinder S, Rosalind S, Mallina S, Gurdeep S. Management of Sinonasal Inverted Papillomas: Endoscopic Medial
Maxillectomy. Med J Malaysia. 2008 Mar;63(1):58-60

18. Berenholz L, Kessler A, Sarfati S, Eviatar E, Segal S. Chronic Sinusitis: A sequela of Inferior Turbinectomy. Am J Rhinol. 1998;12(4):257-261.

19. Ly TH, deShazo RD, Olivier J, Stringer SP, Daley w, Stodard CM. Diagnostic Criteria for Atrophic Rhinosinusitis. Am J Med. 2009 Aug;122(8):747-753.

20. Hildenbrand T, Weber RK, Brehmer D. Rhinitis Sicca, dry nose and Atrophic rhinitis: a review of the literature. Eur Arch Otorhinolaryngology. 2011;268:17-26.

21. Sozansky J, Houser S. Pathophysiology of empty nose syndrome. Laryngoscope. 2015 Jan;125(1):70-4.

Dr. Taseer Feroze Din

Division of Otorhinolaryngology University of Cape Town $\mathrm{H}-53$ Old Main Building Groote Schuur Hospital Observatory Cape Town 7925 South Africa

Tel: +27726934080

E-mail: taseer_d4@hotmail.com

ISSN: 2589-5613 / @2020 The Author(s). This work is licensed under a Creative Commons Attribution 4.0 International License. The images or other third party material in this article are included in the article's Creative Commons license, unless indicated otherwise in the credit line; if the material is not included under the Creative Commons license, users will need to obtain permission from the license holder to reproduce the material. To view a copy of this license, visit http://creativecommons.org/ licenses/by/4.0/ 